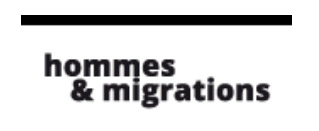

\section{Hommes \& migrations}

Revue française de référence sur les dynamiques

migratoires

1308 | 2014

Les Paris des migrants

\title{
L'immigration, une source de vitalité pour la ville de Paris
}

Entretien avec Colombe Brossel, adjointe à la maire de Paris en charge de la sécurité, de la prévention, de la politique de la Ville et de l'intégration

\section{Marie Poinsot}

\section{(2) OpenEdition}

Journals

\section{Édition électronique}

URL : http://journals.openedition.org/hommesmigrations/2990

DOI : 10.4000/hommesmigrations.2990

ISSN : 2262-3353

Éditeur

Musée national de l'histoire de l'immigration

\section{Édition imprimée}

Date de publication : 1 octobre 2014

Pagination : 28-30

ISBN : 978-2-919040-29-2

ISSN : 1142-852X

Référence électronique

Marie Poinsot, «L'immigration, une source de vitalité pour la ville de Paris », Hommes \& migrations [En ligne], 1308 | 2014, mis en ligne le 01 octobre 2017, consulté le 30 avril 2019. URL : http://

journals.openedition.org/hommesmigrations/2990; DOI : 10.4000/hommesmigrations.2990 


\section{L'IMMIGRATION, UNE SOURCE DE VITALITÉ POUR LA VILLE DE PARIS}

Entretien avec COLOMBE BROSSEL, adjointe à la maire de Paris en charge de la sécurité, de la prévention, de la politique de la Ville et de l'intégration

Hommes \& Migrations : Paris reconnaît-il les Parisiens venus d'ailleurs dans son histoire qu'ils ont contribué à construire?

Colombe Brossel : Notre ville s'est construite grâce à l'arrivée de Parisiens nés ailleurs, en France ou à l'étranger, qui ont contribué à développer son dynamisme culturel, économique et démocratique. Ces migrations successives font donc partie intégrante de notre histoire et la reconnaissance de l'apport de l'immigration pour notre ville est indispensable pour permettre aux immigrés ainsi quà leurs descendants de prendre leur place dans la communauté nationale. La valorisation de cette histoire permet également de déconstruire les préjugés sur les immigrés en modifiant le regard porté sur eux par la société.

C'est pourquoi la Ville de Paris mène une politique ambitieuse de valorisation et de démocratisation de la mémoire, de l'histoire et de la culture de l'immigration, par le biais d'expositions, de manifestations culturelles ou du soutien aux associations qui recueillent les archives de l'immigration. Par ailleurs, un travail avec des associations qui mettent en valeur la mémoire privée des habitants grâce à des ateliers d'écriture, des créations de pièces de théâtre, des projets audiovisuels, permet aux Parisiens eux-mêmes de participer à la construction de cette mémoire et à la transmission de leur propre parcours migratoire.

$H \& M$ : Dans la comparaison entre les grandes capitales européennes, est-ce que Paris est une ville singulière dans sa manière d'accueillir les talents étrangers, quels qu'ils soient ?

C. B. : Au $1^{\text {er }}$ janvier 2009, Paris compte 335429 étrangers, dont près de $70 \%$ de ressortissants de pays non communautaires. Environ $20 \%$ de sa population est immigrée ${ }^{1}$.

La maire de Paris a toujours affirmé que tous les résidents de la capitale sont des Parisiens à part entière, quelles que soient leur origine ou leur situation administrative : toutes les personnes habitant Paris, qu'elles soient étrangères ou françaises, en situation régulière ou irrégulière, sont parisiennes et sont toutes considérées avec la même dignité.

Ainsi, la Ville intervient pour aider les migrants à se saisir de leurs droits en les orientant et en les accompagnant. L'objectif est de faciliter l'accès des étrangers aux services de droit commun sans leur imposer de gommer leur culture, leur histoire ou 
leur origine, mais en les intégrant à la vie parisienne. Dépasser la barrière de la langue est le premier pas vers le droit commun et la Ville de Paris consacre plus de 6 millions d'euros à l'apprentissage du français. Pour accompagner les Parisiens ne maîtrisant pas encore suffisamment le français, un guide, traduit en 7 langues, présente les démarches, les loisirs, les possibilités qui s'offrent aux migrants dans la ville. La municipalité travaille également, en lien avec les nombreuses associations parisiennes qui œuvrent sur le terrain, à l'accompagnement et l'accueil des étrangers.

Par ailleurs, pour améliorer l'accueil dans les services de la Ville, une formation spécifique est dispensée aux agents pour les sensibiliser aux parcours migratoires, aux pays d'origine et aux cultures des nouveaux venus.

Paris accueille également, comme d'autres grandes villes internationales, des réfugiés qui arrivent au gré des grandes crises mondiales. La capitale se mobilise pour qu'ils y trouvent la protection nécessaire. La maire de Paris va signer un grand pacte parisien de lutte contre l'exclusion, avec de nombreux partenaires publics et privés, pour combattre la pauvreté et permettre à notre ville de jouer pleinement son rôle de ville refuge.

$H \& M$ : Certains quartiers affichent la visibilité de certaines populations migrantes dans leurs espaces commerciaux (XIII ${ }^{\mathrm{e}}$, Belleville, La Chapelle, Strasbourg-Saint-Denis, Château-d'Eau, etc.) ? Ces "centralités commerciales" récentes sont-elles appréciées et reconnues par la Ville de Paris?

C. B. : La Ville n'a pas la volonté de valoriser la dimension ethnique de certains quartiers ni de favoriser les mono-commerces. Toutefois, les nombreux talents et initiatives qui se développent dans les quartiers populaires doivent être soutenus. Des personnes étrangères ou migrantes, plus nombreuses dans ces quartiers, participent à cette dynamique et leur apport à la vitalité économique de notre ville est indéniable et doit être accompa- gné. C'est, par exemple, le cas du projet mode à la Goutte-d'Or qui s'emploie à structurer les nombreux et talentueux acteurs de la filière mode dans ce quartier, par une mise en réseau, des formations, notamment en français, et une aide à la structuration de la filière.

$H \& M$ : Pensez-vous que Paris permet des parcours de formation et de réussite professionnelles pour les immigrés et leurs descendants? C. B. : Paris met en place et soutient de nombreux parcours de formation à destination des Parisiens les plus fragiles, notamment ceux des quartiers populaires. Bien évidemment, les personnes migrantes ont accès à toutes ces formations mais aucune ne leur est spécifiquement réservée. Le choix du public bénéficiaire des formations ne peut en effet se faire sur la qualité de migrant, la nationalité ou l'origine. Les formations sont construites autour des besoins des personnes et des quartiers. Par ce biais, la Ville de Paris tâche d'aider les migrants dans leur recherche d'emploi et dans leur formation professionnelle. Par exemple, des formations spécifiques, intitulées "passerelles linguistiques vers l'emploi", allient 500 heures par an de formation linguistique et 600 heures par an de formation professionnelle.

\section{$H \& M$ : Est-ce que se manifestent à Paris des ten-} sions ou des conflits entre populations étrangères cohabitant dans les mêmes quartiers ?

C. B. : Les discriminations et stéréotypes concernent tous les Parisiens, quelles que soient leurs origines. À l'instar des autres Parisiens, les immigrés peuvent être tout à la fois victimes et auteurs de discriminations.

Pour autant, les immigrés continuent à être plus stigmatisés et discriminés et nombreux sont ceux qui vivent dans une situation sociale fragile. Cette réalité est inacceptable et la Ville de Paris conduit de nombreuses actions de lutte contre les discriminations, notamment celles liées à l'origine. La maire de Paris a souhaité qu'un plan parisien de lutte 
contre les discriminations soit élaboré pour que ce combat infuse toutes les politiques menées à Paris. Par ailleurs, les services de la Ville et les associations parisiennes, par le travail de lien social qu'ils mènent au quotidien au plus près des territoires, contribuent à tisser des relations entre habitants d'horizons divers.

H\&M : Comment la Ville de Paris peut-elle transformer l'image des immigrés, résidents et acteurs du développement dela Ville? Par quelles démarches culturelles ou pédagogiques ?

C. B. : La crise économique, mais aussi politique et de sens, que connaît l'Europe actuellement entraîne une augmentation des discours anti-immigration, les dernières élections européennes nous l'ont montré.

Pourtant, toutes les données objectives prouvent que l'immigration est économiquement positive pour les pays receveurs. De plus, les immigrés travaillent le plus souvent dans des secteurs sous tension (bâtiment, service à la personne).

La Ville de Paris s'attache, par la valorisation de l'histoire et de l'apport culturel des migrants, à changer les perceptions. Il nous faut désormais affirmer plus fermement l'apport des migrants à la vitalité et au développement économiques de notre ville. Nous avons d'ailleurs demandé à l'Atelier parisien d'urbanisme (Apur) de mener une étude sur la question.

$H \& M$ : La participation des étrangers à la vie municipale et associative est-elleactive? Quelles sont les initiatives de la Ville de Paris pour encourager la citoyenneté des Parisiens étrangers?

C. B. : La participation à la vie citoyenne des étrangers, et plus particulièrement des citoyens extracommunautaires qui n'ont pas encore obtenu le droit de vote aux élections locales, est indispensable à la vie démocratique parisienne.

Les Parisiens étrangers peuvent s'impliquer, comme tous les autres habitants, dans la vie parisienne à travers les associations et les instances représentatives. Ils sont d'ailleurs nombreux dans les associations, les conseils d'école ou les conseils de quartier. Pour favoriser et développer la participation de tous les Parisiens, indépendamment de leur âge, de leur situation administrative ou de leur nationalité, la maire de Paris a annoncé la création d'une "carte du citoyenne de Paris" qui marquera l'appartenance à la Ville. Elle permettra également à tous de poser des actes citoyens comme voter pour le budget participatif par exemple.

$H \& M$ : Dans les projets de constitution du Grand Paris, la problématique migratoire (répartition géographique des flux, politique d'accueil des nouveaux arrivants, approches interculturelles dans les quartiers) est-elle intégrée comme une dimension importante des défis à relever dans cette redéfinition de l'échelle territoriale de la métropole?

C. B. : La Ville de Paris s'attache à créer des liens avec les communes voisines. La métropole du Grand Paris va permettre de structurer, d'étendre et de densifier ces partenariats et coopérations. Les immigrés arrivent le plus souvent dans les zones fortement urbanisées qui leur paraissent plus riches en opportunités. De ce fait, plus que dans les territoires ruraux, les métropoles auront à prendre en compte ces flux de façon concertée et harmonisée. Le mouvement est déjà en route et ne pourra que s'amplifier. 